\title{
Introduction to Special Issue on Liver Transplant and HCC at Inonu University, Turkey
}

\author{
Sezai Yilmaz ${ }^{1} \cdot$ Brian I. Carr ${ }^{1}$ \\ Published online: 27 August 2020 \\ (C) Springer Science+Business Media, LLC, part of Springer Nature 2020
}

Inonu University Turgut Ozal Medical Center in Malatya, central Turkey was established in 1996. Liver transplantation was established there 18 years ago by Vedat Kirimlioglu, and by Sezai Yilmaz, who directs the Liver Transplant Institute, which opened as a separate building on the Medical Center grounds in 2016 as the world's first dedicated Liver Transplant Institute and Hospital. During that time, more than 3000 liver transplants have been performed, of which over 400 were for hepatocellular carcinoma (HCC). HCC transplants are averaging about 40 per year. We now perform the largest number of living donor liver transplants in Turkey and for any center in Europe. In June 2019, we performed five simultaneous living donor liver transplantations including one pediatric and four adult patients. This shows that we have accumulated considerable experience. Experienced centers have the personnel, equipment, and facilities to perform multiple simultaneous procedures and need to perform multiple simultaneous procedures under needed conditions, such as planned and emergency liver transplantations and "swap" liver transplants. This may reflect the greater than 75\% 5-year survival rate for patients with HCC and the relatively low cost (\$50,000 compared with approximately $\$ 550,000$ in the USA). Furthermore, our liver transplant program is based predominantly on the use of live donors, as a result of which, there is minimal time to wait for the surgery. As a result, patients and their families come from across the Middle East and Central Asia. As a fringe benefit, Malatya province provides $80 \%$ of the world's apricots.

Due to advances in recent years in expansion of the criteria for liver transplant for HCC (more patients with larger tumors can be candidates), progress in identifying patient subsets with better prognosis (small tumors, low serum alpha-fetoprotein, low serum gamma glutamyl transpeptidase levels) and worse prognosis (presence of portal vein thrombosis), use of downstaging techniques to permit transplant for patients with larger tumors, the possibilities of expanding the donor pool using xenotransplantation, approaches to treating portal vein thrombosis, identification of psychological issues in patients and liver donors, it was deemed useful to provide a collection of essays and studies from our center in the current issue, that cover matters in current progress and problems in the subject.

Publisher's Note Springer Nature remains neutral with regard to jurisdictional claims in published maps and institutional affiliations.
Sezai Yilmaz

sezai.yilmaz@inonu.edu.tr

Brian I. Carr

brianicarr@hotmail.com

1 Liver Transplant Institute, Inonu University, Malatya, Turkey 